\title{
Justification of Special Population
}

National Cancer Institute

\section{Source}

National Cancer Institute. Justification of Special Population. NCI Thesaurus. Code

C161318.

The explanation or reason as to why a special population of subjects is considered for inclusion in a clinical study. 